\title{
Sindrome de muerte súbita del lactante: ¿cohabitación sí, colecho no?
}

\section{Objetivo}

Detectar los factores de riesgo asociados al síndrome de muerte súbita del lactante (SMSL).

\section{Diseño}

Síntesis de varios estudios caso control de acuerdo al protocolo de Acción Concertada de la Unión Europea en SMSL.

Lugar

20 centros de Europa (seis de Europa oriental) desde 1992 a 1996.

\section{Participantes}

Se incluyeron 745 casos de SMSL. Sólo fueron incluidos los casos a los que se les había realizado autopsia, consistiendo en promedio en el 78\% (rango intercuartilo* 64-91\%) de los casos registrados de SMSL en el área cubierta por cada centro durante el periodo de cada estudio. 2411 controles vivos.

\section{Evaluación de factores de riesgo}

Para cada caso se tomaron dos o más controles de la misma edad y lugar de residencia. Se realizaron cuestionarios similares a ambos grupos. Para evitar el sesgo de recuerdo*, las preguntas sobre los momentos finales de los casos fueron reemplazadas en los controles por preguntas correspondientes a la misma hora pero del día previo a la entrevista. Se realizó un análisis unifactorial y multivariable, calculando OR e IC 95\%.

\section{Medición de resultados principales}

SMSL fue definido como la muerte súbita e inesperada de un lactante luego del período neonatal que no puede ser explicada por la historia clínica, por detalles de las circunstancias de la muerte, ni por una autopsia realizada bajo protocolo internacional.

\section{Resultados principales}

La incidencia de SMSL varió de 0,17 por mil en Hungría a 1,3 por mil en Alemania (mediana 0,6; rango intercuartilo*: 0,4 - -0,7). Fue mayor en invierno y en varones $(63 \%)$. La edad pico fue a las diez semanas, $7,77 \%$ fueron menores de un mes y $18 \%$, mayores de seis meses. Los resultados del análisis de los factores de riesgo se resume en el cuadro 1.

\section{Conclusión}

Los factores de riesgo evitables en la preparación para el sueño de los bebes sugieren una base para una reducción adicional importante de las tasas de incidencia de SMSL.

Fuente de financiamiento: Foundation for study of infant Deaths, London school of Hygiene and Tropical medicine.

Cuadro 1: resultados del análisis multivariable de los factores de riesgo de síndrome de muerte súbita del lactante.

\begin{tabular}{|c|c|c|c|c|}
\hline \multicolumn{2}{|c|}{ Factor de riesgo } & Casos & Gontroles & OR (IC95\%) \\
\hline \multirow{2}{*}{$\begin{array}{l}\text { Posición en la que había } \\
\text { sido dejado }\end{array}$} & De costado vs. supino & 250 & 894 & $1,31(0,93--1,81)$ \\
\hline & Prono vs. supino & 268 & 269 & $13,1(8,51 \cdots-20,2)$ \\
\hline \multirow{5}{*}{$\begin{array}{l}\text { Tabaquismo materno } \\
\text { y/o colecho }\end{array}$} & No fuma, no colecho & 249 & 1621 & 1 \\
\hline & No fuma, si colecho & 32 & 139 & $1,56(0,91--2,68)$ \\
\hline & Menos de diez cig./día, no colecho & 133 & 328 & $1,52(1,10--2,09)$ \\
\hline & Más de diez cig./día, no colecho & 194 & 247 & $2,43(1,76--3,36)$ \\
\hline & Fuma, si colecho & 111 & 56 & $17,7(10,3--30,3)$ \\
\hline \multicolumn{2}{|c|}{ Uso de chupete siempre vs. no uso } & 394 & 1492 & $0,74(0,58-0,95)$ \\
\hline \multicolumn{2}{|c|}{ Historia de episodio de amenaza aparente de la vida (ALTE) } & 79 & 73 & $2,76(1,76--4,32)$ \\
\hline \multicolumn{2}{|c|}{ Nacimiento múltiple vs. único } & 47 & 36 & $2,40(1,27 \cdots-4,52)$ \\
\hline \multirow[t]{3}{*}{ Peso de nacimiento } & 2500 a $3499 g$ & 381 & 1131 & $1,44(1,14--1,83)$ \\
\hline & 2000 a $2499 \mathrm{~g}$ & 84 & 59 & $3,36(1,92--5,88)$ \\
\hline & Menor a $2000 \mathrm{~g}$ & 61 & 28 & $4,83(2,36--9,88)$ \\
\hline \multirow[t]{4}{*}{ Edad materna (años) } & 26 a 30 & 224 & 875 & $1,64(1,22--2,21)$ \\
\hline & 21 a 25 & 228 & 529 & $3,21(2,30 \cdots-4,48)$ \\
\hline & 19 a 20 & 75 & 92 & $6,91(4,09-11,7)$ \\
\hline & Menos de 18 & 46 & 31 & $11,0(5,38-22,4)$ \\
\hline \multirow[t]{4}{*}{ Nacidos vivos previos } & 1 & 256 & 862 & $2,76(2,06-3,70)$ \\
\hline & 2 & 161 & 372 & $3,94(2,76--5,63)$ \\
\hline & 3 & 73 & 122 & $4,68(2,89 \cdots-7,58)$ \\
\hline & 40 más & 50 & 50 & $10,6(5,78 \cdots-19,3)$ \\
\hline \multirow{2}{*}{$\begin{array}{l}\text { Consumo de alcohol las } \\
\text { ultimas } 24 \mathrm{~h}\end{array}$} & Uno a dos vasos vs. nada & 50 & 193 & $1,00(0,63--1,57)$ \\
\hline & Tres 0 más vasos vs. nada & 39 & 53 & $2,33(1,28--4,21)$ \\
\hline \multirow[t]{2}{*}{ Cohabitación sin colecho } & Usualmente ${ }^{a_{*}}$ & 180 & 816 & $0,48(0,34--0,69)$ \\
\hline & En la última ocasión ${ }^{a}$ & 93 & 474 & $0,32(0,19--0,55)$ \\
\hline \multicolumn{2}{|c|}{ Acolchado usado en la última ocasión ${ }^{\mathrm{a} *}$} & 191 & 375 & $1,82(1,30--2,58)$ \\
\hline \multicolumn{2}{|c|}{ Bebe hallado con la cabeza cubierta ${ }^{a}$} & 154 & 69 & $12,5(6,47$ a $-24,1)$ \\
\hline
\end{tabular}

a- Factores de riesgo no evaluado en todos los centros. 


\section{Comentario}

El SMSL es la muerte inesperada de un niño que se encontraba aparentemente sano hasta el momento de acostarlo,2. El $80 \%$ ocurre antes de los seis meses y es raro antes del primer mes de vida.

En los países desarrollados, y a pesar de la gran reducción del SMSL por las campañas para colocar a los niños en decúbito supino $^{3-4}$, sigue siendo la primera causa de mortalidad infantil luego de la primera semana de vida.

En Argentina, para 2001 era la tercera causa de muerte luego de las lesiones no intencionales (ex accidentes) y las causas respiratorias $^{5 .}$ Sin embargo, es difícil conocer su real frecuencia $(0,58$ por mil para el año 2002) ${ }^{6}$ ya que en la mayoría de los casos no se realiza autopsia, debido a que a muchas de las muertes sin causa aparente se las suele rotular como "paro cardiorrespiratorio no traumático"6.

Este reporte resume varios estudios de casos y controles, el diseño más apropiado para estudiar los determinantes de SMSL, ya que esta metodología permite analizar varios factores de riesgo a la vez y es la de elección cuando se trata de investigar la asociación de determinantes que no pueden ser controlados por el investigador (ej. asignados al azar) respecto de eventos poco frecuentes como el SMSL.

El hallazgo de un claro patrón "dosis-respuesta" y de OR elevados (ver cuadro 1) refuerza la probabilidad de que exista una vinculación causal, y alienta la esperanza de que conociendo las causas se pueda evitar el desenlace. Pero, lamentablemente varios de los factores de riesgo detectados son "estructurales" y no serían pasibles de reducción a través de la educación de los padres o cuidadores. Mencionamos entre estos al bajo peso al nacer, etnia, sexo masculino, prematurez, embarazo múltiples, madre adolescente, multiparidad, edad del fallecimiento (dos a tres meses) y la mayor frecuencia invernal. ${ }^{8}$

De los factores de riesgo modificables los más significativos fueron "el cambio de la posición lateral a la posición prona", el "decúbito prono" y el "colecho con madre fumadora".

$\mathrm{Si}$ bien, la posición en decúbito lateral suele ser indicada como alternativa a la de decúbito supino, es menos estable. Por ejemplo, en este trabajo $12 \%$ de los niños fallecidos habían sido acostados de costado y hallados boca abajo (OR para el cambio de posición 45,4; IC95: 23,4-87).

En hijos de madre fumadora, el colecho (definido como dormir toda la noche con un adulto) se asoció a un alto riesgo de SMSL, especialmente durante las primeras semanas de vida (OR a las dos semanas: 27; IC95: 13,3-54,9); mientras que fue menor en hijos de madres no fumadoras (OR a las dos semanas de vida: 2,4; IC95: $1,2-4,6)$ y sólo durante las primeras ocho semanas de vida. Es interesante destacar los resultados del estudio de Blair y col., que muestra que los bebes que comparten la cama con los padres y luego son dejados boca arriba en sus cunas no tienen riesgo aumentado de $\mathrm{SMSL}^{9}$, hecho de importancia no menor ya que el colecho suele ser aconsejado para fomentar la lactancia materna $^{10,11}$, si bien hay controversias al respecto ${ }^{12}$.

Dormir en la misma habitación que los padres, pero no en la misma cama, es un factor protector.

Cabe destacar que $52 \%$ de los casos de SMSL podrían haberse prevenido haciendo dormir a los niños en la misma habitación pero no en el mismo lecho, conclusión que concuerda con otros trabajos ${ }^{9,13,14}$.

\section{Conclusiones de los comentadores}

Para disminuir el riesgo de SMSL, existe evidencia observacional para recomendar que el bebé duerma boca arriba y en la misma habitación que los padres pero no en el mismo lecho (en una cuna separada). Las madres fumadoras deberían recibir el consejo de evitar dormir toda la noche con el bebe.

Juan Pablo Mouesca [ Pediatra, Monte Grande.]

Leandro Castillo [ Jefe de Residentes de Pediatría del Hospital. de Niños R.Gutiérrez. ]

Mouesca J, Castillo L. Síndrome de muerte súbita del lactante: ¿cohabitación sí, colecho no? Evid. actual. pract. ambul. 2005;8:100-101. Comentado de: Carpenter R, Irgens L, Blair P, England P, Fleming P, Huber J, Jorch G, Schreuder P Sudden unexplained infant death in 20 regions in Europe: case control study. The Lancet - Vol. 363, Issue 9404, 17 January 2004, Pages 185-191. PMID: 14738790

\section{Referencias}

1. Rocca Rivarola M. Síndrome de muerte súbita del lactante. En Macri C, Teper A. Enfermedades respiratorias pediátricas. McGraw-Hill Interamericana 2003;67:647-654

2. Las Heras Boneto J. Muerte súbita en el lactante. En Pediatria Meneghello $5^{\mathrm{a}}$ Ed. Eds. Meneghello J, Fanta E,Paris E, Puga T. Editorial Medica Panamericana 1997; 477:2693-5.

3. Farrel P. Weiner G, Lemons J. Síndrome de muerte súbita del lactante, episodio de aparente amenaza a la vida, apnea y uso de monitores domésticos. PIR(esp) 2002;23:129-135.

4. Rusen I, Liu S, Sauve R, et al. Sudden infant death syndrome in Canada: Trends in rates and risk factors, 1985-1987. Chronic Dis Can. 2004; 25:1-6.

5. SAP-UNICEF. Salud materno-infanto-juvenil en cifras 2003. pg 24.

6. Ministerio de Salud. Dirección de Maternidad e infancia. Actualización Sindrome de muerte súbita del lactante.

7. Kraus HF. The international standardized autopsy protocol for sudden and unexpected infant death. Scandinavian University Press, $1994: 81-95$.

8. Daley KC. Update on sudden infant death syndrome. Curr Opin Pediatr. 2004 Apr;16 (2):227-32.

9. Blair P, Fleming P, Smith I et al. Babies sleeping with parents: case-control study of factors influencing the risk of the sudden infant death syndrome. CESDI SUDI research group. BMJ 1999;319:1457-61

10. UNICEF. Facts for life breastfeeding Apr 2002. www.unicef.org/ffl/04/3.htm

11. Ball $\mathrm{H}$. breastfeeding, bed-sharing, and infant sleep. Birth 2003:30:181-8.

12. Jenik A, Ceriani Cernadas J. La alimentación a pecho como factor de prevención del síndrome de muerte súbita del lactante: acuerdos y controversias. Arch.argent.pediatr 2004;102:277-286

13. Scragg R, Mitchell E, Stewart A et al. Infant room-sharing and prone sleep position in sudden infant death syndrome. New Zealand Cot Death Study Group. Lancet 1996;347:7-12. 14. Gessner B, Ives G, Perham-Hester K. Association between sudden infant death syndrome and prone sleep position, bed sharing, and sleeping outside an infant crib in Alaska. Pediatrics 2001;108:923-7. 